\title{
Synthesis and Characterization of Janus Magnetic Nanoparticles and its Application as an Adsorbent
}

\author{
Zayed Bin Zakir Shawon \\ Department of Chemical and Biomolecular Engineering \\ National University of Singapore, 4 Engineering Drive 4, Singapore 117576 \\ Tel: +65 6516 2099, Fax: +65 67791934
}

\begin{abstract}
A simple and quick method to synthesize Janus magnetic particles in the nano-sized range was conducted. Magnetic nanoparticles (MNPs) with diameters in the range of 10-15 $\mathrm{nm}$ were prepared by the co-precipitation of $\mathrm{Fe}^{2+}$ and $\mathrm{Fe}^{3+}$ precursors under alkaline and inert condition. The Pickering Emulsion method was used for the immobilization of magnetic nanoparticles onto the surface of paraffin wax balls via water-molten wax emulsion. Silanization of the exposed side of the immobilized magnetic particles was carried out with APTES and the waxes were subsequently dissolved to obtain free Janus nanoparticles. Characterization tests such as TGA, SEM-EDX, TEM and FTIR were performed to ascertain the partial coating of the Janus nanoparticles. Moreover, adsorption capacity of the Janus nanoparticles on $\mathrm{Hg}^{2+}$ ions was found to be intermediate between those using the uncoated and fully coated nanoparticles indicating successful synthesis of Janus nanoparticles.
\end{abstract}

\section{Introduction}

An emerging interest in recent years is the development of asymmetrical microparticles and nanoparticles, where the surface chemistry functionality differs for each of the two sides of the particle. These kinds of particles have been dubbed as Janus particle. The attraction for the creation and usage of Janus particles is high-large dipole moments can be generated on a single particle by surface functionalization of particle with opposite charges on either side for usage as electrorheological fluid ${ }^{8}$; self-assembly characteristics may be conferred to the particles if there are both hydrophilic and hydrophobic sides on the particles. Several methods have been developed to produce macro or micro sized Janus particles but, nano sized Janus particles synthesis is still challenging ${ }^{9,13}$.

In this article, we have presented a method to produce Janus nanoparticles (10-15 $\mathrm{nm}$ ) directly by adopting the Pickering Emulsion method ${ }^{9}$. This method is favorable as compared to many other existing methods, as it poses a direct synthesis path instead of using additional nanoparticles as masking particles in the case of heterodimers $^{11,12}$ or doing extra coating on the particles which will inevitably increase the overall size $^{7}$. Furthermore, this method is also advantageous in terms of shorter synthesis time. Moreover, we have used these particles to adsorb $\mathrm{Hg}^{2+}$ ions efficiently.

\section{Methods}

\subsection{Materials}

Iron (II) chloride tetrahydrate (98\%), Alfa Aesar; Iron (III) chloride hexahydrate (98\%), Fluka; Ammonium

\footnotetext{
*Corresponding Author: Zayed Bin Zakir Shawon

E-mail: shawon@nus.edu.sg
}

hydroxide $(25 \% \quad \mathrm{v} / \mathrm{v}), \quad$ Merck; Aminopropyl triethoxysilane (APTES) 98\%, Alfa Aesar; Methanol (AR grade), Merck; Paraffin (57 to $60^{\circ} \mathrm{C}$ non-caking solidification point), Merck; Chloroform, Merck.

\subsection{Synthesis of bare magnetic nanoparticles}

Magnetic nanoparticles were prepared via the wellestablished co-precipitation technique using $\mathrm{Fe}^{2+}$ and $\mathrm{Fe}^{3+}$ salts in a molar ratio of $1: 2$ under alkaline condition and inert atmosphere ${ }^{1,5,10}$. $0.86 \mathrm{~g}$ of $\mathrm{FeCl}_{2} \cdot 4 \mathrm{H}_{2} \mathrm{O}$ and $2.35 \mathrm{~g}$ of $\mathrm{FeCl}_{3} \cdot 6 \mathrm{H}_{2} \mathrm{O}$ were dissolved in $40 \mathrm{ml}$ of ultrapure water under inert atmosphere by bubbling nitrogen through the solution. The solution was subsequently heated to $80^{\circ} \mathrm{C}$ with vigorous stirring at $1000 \mathrm{rpm}$ by bench stirrer. $5 \mathrm{ml} \mathrm{NH}_{4} \mathrm{OH}$ was then added to the solution after solution was heated to $80{ }^{\circ} \mathrm{C}$. An aging time of $30 \mathrm{~min}$ was chosen to achieve the desired MNP size distribution and to ensure the complete precipitation of $\mathrm{Fe}_{3} \mathrm{O}_{4}$ magnetic nanoparticles. The reaction yields approximately $1 \mathrm{~g}$ of $\mathrm{Fe}_{3} \mathrm{O}_{4}$, based on the following chemical reaction:

$\mathrm{FeCl}_{2}(\mathrm{aq})+2 \mathrm{FeCl}_{3}(\mathrm{aq})+8 \mathrm{NH}_{4} \mathrm{OH} \rightarrow \mathrm{Fe}_{3} \mathrm{O}_{4}(\mathrm{~s})+$ $8 \mathrm{NH}_{4} \mathrm{OH}(\mathrm{aq})+4 \mathrm{H}_{2} \mathrm{O}(\mathrm{l})$

\subsection{Synthesis of Janus nanoparticles}

The Janus APTES nanoparticles were produced using the Pickering Emulsion technique 9 . These emulsions are thermodynamically stable ${ }^{6}$. It was found that the freeze-dried nanoparticles did not serve to stabilize the emulsion, with the two distinct wax and water phase forming upon cooling. This can be attributed to the lost of surface functional group on the nanoparticles, resulting in a lowering of wet-ability of the particles with the wax balls. Therefore, wet bare magnetic 


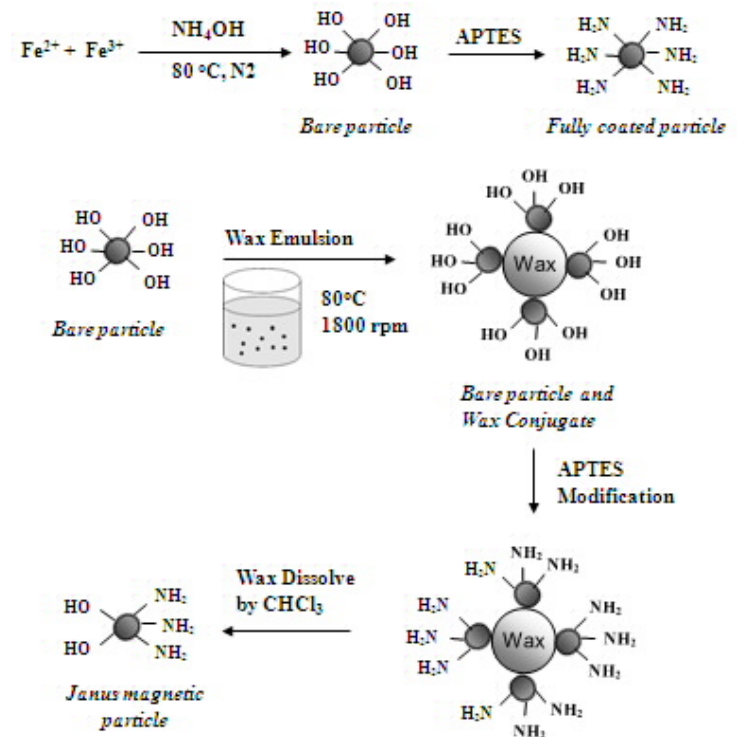

Scheme 1. Reaction steps to prepare bare magnetite particles and to coat with APTES fully or partially

nanoparticles were used to stabilize the wax-water emulsion by forming a monolayer on the dispersed wax phase, as it lowers the overall surface energy of the system.

Firstly, $0.2 \mathrm{~g}$ of wet bare magnetic nanoparticles were dispersed homogeneously in $40 \mathrm{ml}$ of ultrapure water by means of a sonicator (ultrasonic bath) for $30 \mathrm{~min}$. Secondly, the mixture was then heated to $80^{\circ} \mathrm{C}$ using a hot water bath, after which $5 \mathrm{~g}$ of paraffin wax was added to the mixture and allowed to melt. A bench stirrer was utilized to provide mechanical stirring at $1950 \mathrm{rpm}$ for $45 \mathrm{~min}$ to disperse the wax phase in the continuous water phase. The resulting nanoparticlesstabilized emulsion was then allowed to cool to room temperature, enabling the freezing of the wax balls and the immobilization of the magnetic nanoparticles on its surface. The wax balls were filtered and washed repeatedly with ultrapure water to wash off nanoparticles that were either unattached or weakly attached, and were air-dried for 10 hours.

The wax balls were then placed in $100 \mathrm{ml}$ of $2.1 \mathrm{mM}$ APTES in methanol solution, and silanization of the exposed surface of the exposed surface of the nanoparticles were carried out for $1 \mathrm{hr}$. Pure methanol was used to wash the wax balls after filtration in order to remove excess APTES, and subsequently the wax balls were air-dried for 2 hrs. The Janus magnetic nanoparticles were released by the dissolution of the wax balls in excess chloroform, and magnetic decantation was done to obtain the Janus particles from the wax-chloroform solution.

\section{Particle Characterization}

\subsection{Scanning Electron Microscopy and Energy Dispersive X-ray Analysis}

SEM images of the wax balls are as shown in Fig. 1a, assuming an almost spherical shape with an average diameter of $500 \mu \mathrm{m}$. Fig. 1a provides a close-up on the surface of the wax balls, showing high coverage by the magnetic nanoparticles of up to $77 \%$ of the surface on some of the wax balls.
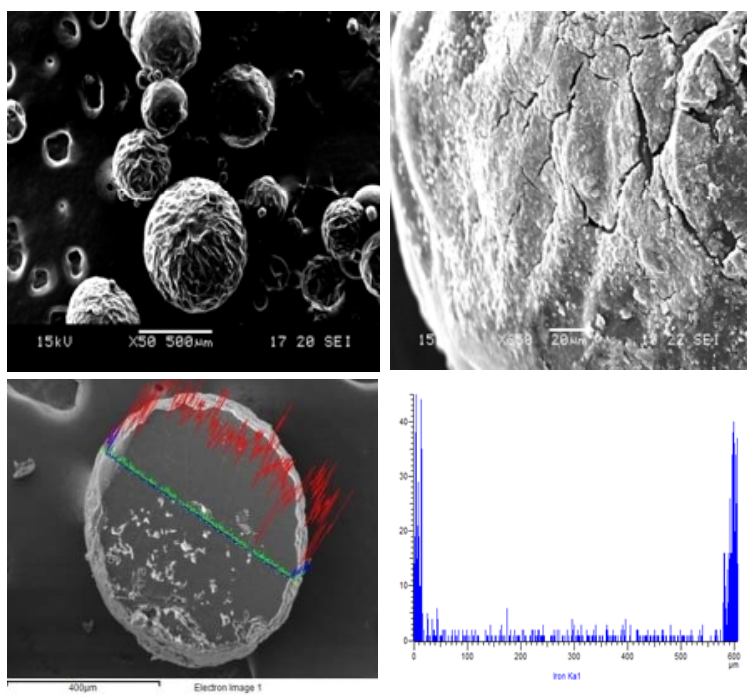

Fig.1. (a) SEM image of wax balls after filtration, (b) EDX along the cross-section of a cut wax balls.

The results for the EDX analysis of the distribution of nanoparticles on the wax balls are as illustrated in Fig. $1 \mathrm{~b}$, which proved conclusively that majority of the magnetic nanoparticles are located on the surface of the wax-water interface instead of within the wax balls. Furthermore, EDX analysis was done on a layer of Janus nanoparticles and a layer of fully coated APTES nanoparticles, with the results showing that the fully coated APTES nanoparticles have a much higher silicon weight percentage that the Janus nanoparticles. Therefore, it is conclusive that the Janus nanoparticles should have a bare side which resulted in the lower silicon weight percentage as compared to the fully coated nanoparticles.

Table 1. Silicon weight percentage of Janus and fully coated nanoparticles

\begin{tabular}{c|c|c}
\hline Samples & $\begin{array}{c}\text { Janus Nanoparticles } \\
\text { (Silicon wt\%) }\end{array}$ & $\begin{array}{c}\text { Fully coated } \\
\text { Nanoparticles } \\
\text { (Silicon wt\%) }\end{array}$ \\
\hline 1 & 0.40 & 1.53 \\
\hline 2 & 0.49 & 1.59 \\
\hline
\end{tabular}




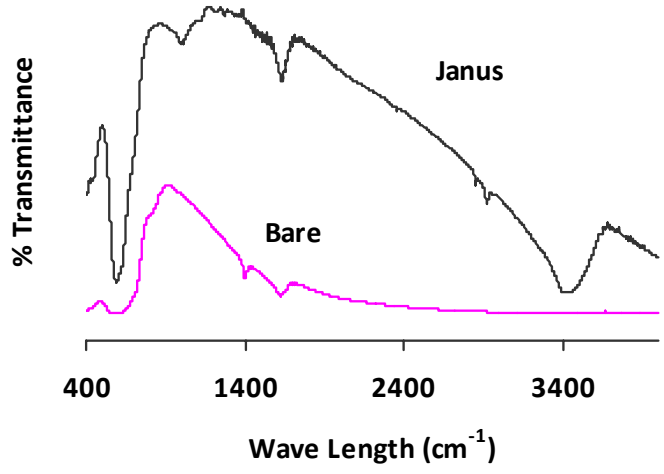

Fig.2. FTIR Spectra of Bare and Janus Magnetic Nanoparticles

\subsection{Fourier Transform Infrared Spectroscopy (FTIR)}

FTIR spectra of the APTES coated Janus nanoparticles is as depicted in Fig. 2. Looking at the spectra, the peak at $590 \mathrm{~cm}^{-1}$ corresponds to $\mathrm{Fe}-\mathrm{O}$ bonding, while the peak at around $1000 \mathrm{~cm}^{-1}$ is indicative of the bonding of Si-O. The appearance of peak at $3410 \mathrm{~cm}^{-1}$ is characteristic of the bending vibration of the $\mathrm{N}-\mathrm{H}$ bond. The occurrences of the peaks provide evidence for the successful silanization of the surface of the Janus magnetic nanoparticles ${ }^{2-5}$.

\subsection{Thermogravimetric Analysis (TGA)}

In order to determine whether the Janus nanoparticles were successfully produced by the method prescribed above, TGA of the bare, Janus and full coated magnetic nanoparticles were conducted and compared in Fig. 3.

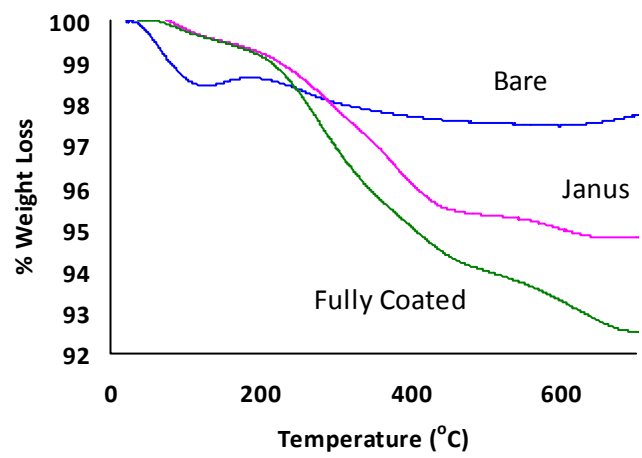

Fig.3. TGA thermograms of bare, Janus and fully coated nanomagnetic particles

The bare nanoparticles incurred a weight loss of approximately $2 \%$ when heated till $800^{\circ} \mathrm{C}$ at ramping rate of $20^{\circ} \mathrm{C} / \mathrm{min}$; the weight loss of the full coated nanoparticles was about $7.5 \%$. This is in agreement with literature value ${ }^{5}$. It can be seen that the weight loss of $5 \%$ for the Janus particles is between the bare and the full coated particles. Furthermore, when the Janus nanoparticles were produced using an APTES concentration that is 200 times higher, the weight loss still remained constant at 5\%. These confirmed the successful synthesis of Janus nanoparticles.

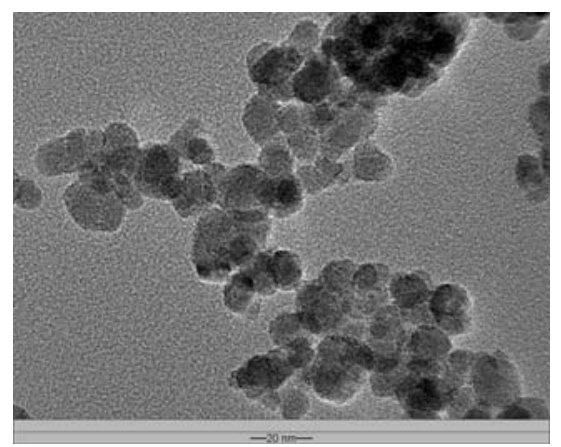

Fig. 4. TEM micrograph of Janus magnetic nano particles. (Scale bar is $20 \mathrm{~nm}$ )

\subsection{Transmission Electron Microscopy (TEM)}

The TEM image of Janus particles is shown in Fig. 4. Since the Janus particles posses dual charge in the same surface it has a tendency to coagulate. The average particle size was found approximately $14-15 \mathrm{~nm}$.

\section{Adsorption Test}

Pollutions occurring due to these types of heavy metals are today a matter concern as they are tremendously harmful to human body as well as to the environment. 'Heavy metal pollution' is also a problem that motivates the development of water treatment technology. Mercury $\left(\mathrm{Hg}^{2+}\right)$ in water resources creates severe environmental and public health problems like kidney and liver failure.

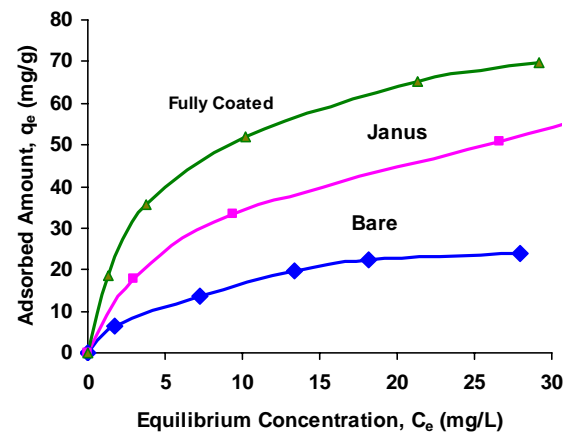

Fig.5. Adsorption isotherms for bare, Janus and fully coated magnetic nanoparticles

In this experiment $\mathrm{Hg}^{2+}$ ions emerging from $\mathrm{HgCl}_{2}$ solution of different concentration ranges were adsorbed onto the surfaces of the nanomagnetic particles. Coating of particles is done to facilitate the adsorbed amount of metal ions onto the surface. 
Adsorption studies were carried out to compare the adsorbed $\mathrm{Hg}^{2+}$ ion quantity by Janus particles with the bare and fully coated particles. Bare particles may show adsorption of metal ions in a smaller quantity but the fully coated particles' adsorption capacity obviously will be higher than that of Janus and bare particles. Adsorption tests were done for five different concentrations of $\mathrm{Hg}^{2+}$ and for all the tests it was found that the adsorbed amount of $\mathrm{Hg}^{2+}$ by Janus particles is always in between than that using bare and fully coated particles. These results also indicate the synthesis of Janus nanomagnetic particles. $\mathrm{Hg}^{2+}$ ion concentration was determined by ICP-MS.

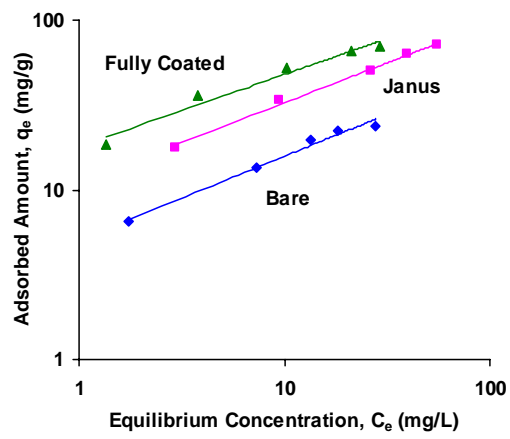

Fig.6. Freundlich plot illustrating the dependences of $q_{e}$ on $C_{e}$ for bare, Janus and fully coated magnetic nanoparticles (logarithmic scale)

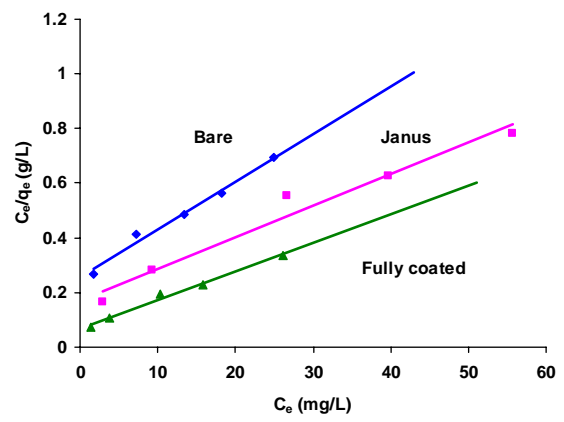

Fig.7. Langmuir plot illustrating the linear dependences of $C_{e} / q_{e}$ on $C_{e}$ for bare, Janus and fully coated magnetic nanoparticles

According to the adsorption isotherms we know that, adsorbed amount $q_{e}$ is dependent on the equilibrium concentration $C_{e}$. This mutual dependence is expressed through the well known Freundlich and Langmuir isotherm models. Linear versions of these two models are popular for the adsorption data fitting and thereby facilitate to derive different adsorption parameters (i.e., adsorption constant $K$, exponent $\mathrm{n}$ and maximum monolayer adsorption $q_{m}$ ).

Langmuir equation is expressed as:

$q_{e}=q_{m} \frac{K_{L} C_{e}}{1+K_{L} C_{e}}$
And its linear form is,

$\frac{C_{e}}{q}=\frac{C_{e}}{q_{m}}+\frac{1}{K q_{m}}$

Freundlich equation is expressed as:

$q_{e}=K_{F} C_{e}^{1 / n}$

And its liner form is,

$\ln \left(q_{e}\right)=\ln K_{F}+(1 / \mathrm{n}) \ln \left(C_{e}\right)$

Fig.6 and Fig.7 show the linear models of Freundlich and Langmuir adsorption isotherms plotted with the experimental data. Different adsorption parameters were derived from these two plots and shown in Table 2. From the regression coefficient $\left(R^{2}\right)$ of these two models, it can be surmised that the experimental data were fitted well with the Freundlich model which indicates multilayer adsorption on the surface.

Table 2. Values of different adsorption parameters

\begin{tabular}{c|c|c|c|c}
\hline Isotherm & $\begin{array}{c}\text { Paramete } \\
\mathrm{r}\end{array}$ & $\begin{array}{c}\text { Bare } \\
\text { Particle }\end{array}$ & $\begin{array}{c}\text { Janus } \\
\text { Particle }\end{array}$ & $\begin{array}{c}\text { Fully } \\
\text { coated } \\
\text { Particle }\end{array}$ \\
\hline \multirow{3}{*}{ Freundlich } & $K_{F}$ & 5.083 & 11.152 & 18.035 \\
\cline { 2 - 5 } & $R^{2}$ & 0.969 & 0.996 & 0.985 \\
\cline { 2 - 5 } & $n$ & 2.031 & 2.142 & 2.354 \\
\hline \multirow{5}{*}{ Langmuir } & $q_{m}(\mathrm{mg} / \mathrm{g})$ & 68.97 & 86.21 & 96.16 \\
\cline { 2 - 5 } & $\begin{array}{c}K_{L} \\
(\mathrm{~L} / \mathrm{mg})\end{array}$ & 0.051 & 0.068 & 0.151 \\
\cline { 2 - 5 } & $R^{2}$ & 0.924 & 0.968 & 0.986 \\
\hline
\end{tabular}

\section{Conclusion}

Magnetic nanoparticles in the size range of $10-15 \mathrm{~nm}$ were prepared by the chemical precipitation method, and subsequently underwent partial surface chemical modification by APTES to produce Janus nanoparticles. Characterization tests were conducted to prove the partial coating of APTES on the Janus nanoparticles. FTIR results confirmed the successful grafting of the functionalized amine group onto the nanoparticles surface. SEM images and EDX analysis shows that the Janus nanoparticles have lower silicon concentration than fully coated nanoparticles, indicative of partial coating of Janus nanoparticles. Furthermore, TGA and adsorption study both gave intermediate results between bare and fully coated nanoparticles, thus proving the successful partial coating on the Janus nanoparticles.

\section{Reference}

1. Peng, Z. G., Hidajat, K., and Uddin, M. S. (2004). Adsorption of bovine serum albumin on nanosized magnetic particles. J. Colloid and Interface Sci. 271: 277-283. 
2. Xu, Z., Liu, Q., and Finch, J. A. (1997). Silanation and stability of 3-aminopropyl triethoxy silane on nanosized superparamagnetic particles: I. Direct silanation. App. Surface Sci. 120: 269-278.

3. Ma, M., Zhang, Y., Yu, W., Shen, H. Y., Zhang, H. Q., and $\mathrm{Gu}, \mathrm{N}$. (2003). Preparation and characterization of magnetite nanoparticles coated by amino silane. Colloids and Surfaces A: Physicochemical. Eng. Aspects. 212: 219-226.

4. Shamim, N., Hong, L., Hidajat, K., and Uddin, M. S. (2007). Thermosensitive polymer (Nisopropylacrylamide) coated nanomagnetic particles: Preparation and characterization. Colloids and Surfaces B: Biointerfaces. 55: 51-58.

5. Tri, P. M., Uddin, M. S., Hidajat, K., and Hong, L. (2009). Adsorption of mercury on nano-magnetic particles surface functionalized by pendant amine. J. Nanosci. Nanotech. 9: 905-908.

6. Sacanna, S., Kegel, W. K., and Philipse, A .P. (2007). Thermodynamically Stable Pickering Emulsions. Phy. Rev. Lett. 98: 158301.

7. Lattuada, M., and Hatton, T. A. (2007). Preparation and Controlled Self-Assembly of Janus Magnetic Nanoparticles. J. Am. Chem. Society. 129: 1287812889.

8. Nakahama, K., Kawaguchi, H., and Fujimoto, K. (2000). A Novel Preparation of Nonsymmetrical
Mircospheres Using the Langmuir-Blodgett Technique. Langmuir. 16: 7882-7886.

9. Hong, L., Jiang, S., and Granick, S. (2006). Simple Method to Produce Janus Colloidal Particles in Large Quantity. Langmuir. 22: 9495-9499.

10. Wu, K. T., Kuo, P. C., Yao, Y. D., and Tsai, E. H. (2001). Magnetic and Optical Properties of $\mathrm{Fe}_{3} \mathrm{O}_{4}$ Nanoparticle Ferrofluids Prepared by Coprecipitation Technique. IEEE Transactions on Magnetics. 37: 2651-2653.

11. Gu, H. W., Yang, Z. M., Gao, J. H., Chang, C. K., and $\mathrm{Xu}, \mathrm{B}$. (2005). Heterodimers of Nanoparticles: Formation at a Liquid-Liquid Interface and Particle-Specific Surface Modification by Functional Molecules. J. Am. Chem. Society. 127: 34-35.

12. Glaser, N., Adams, D. J., Boker, A., and Krausch, G. (2006). Janus Particles at Liquid-Liquid Interfaces. Langmuir. 22: 5227-5229.

13. Cayre, O., Paunov, V. N., and Velev, O. D. (2003). Fabrication of asymmetrically coated colloid particles by microcontact printing techniques. J. Mat. Chem. 13: 2445-2450. 